\title{
23. NON-CIRCULAR MOTIONS IN THE GALAXY AS EXHIBITED BY VERY YOUNG STARS
}

\author{
H. F. WeAver
}

Radio Astronomy Laboratory, University of California, Berkeley

\section{Expansion of the Gaseous and Stellar Components of the Galaxy}

If the gaseous component of the Galaxy is expanding as observed by Rougoor and Oort in the centre of the Galaxy and as postulated by Kerr in his early interpretation of spiral structure, the expansion must represent a phenomenon of fundamental importance in the Galaxy which has, in all probability, been operative for a significant fraction of the age of the Galaxy. Presumably, very young stars formed from this gas and having ages less than $1 \%$ of the age of the Galaxy might be expected to retain in their motions the general character of the large-scale expansion of the gas from which they originated.

\section{The Population of Stars Investigated}

There are available for testing for expansion of the stellar component of the Galaxy 219 stars of type B2 or earlier, 31 clusters and 13 associations having stars of type B2 or earlier. For all these objects radial velocities of quality $a$ or $b$ are available. No recent Lick radial velocities found in the Carnegie General Catalogue of Stellar Radial Velocities, but not elsewhere, are included in this discussion.

\section{The Distance Scale}

For the population of stars described in Section II MK spectral types and luminosity classes are known, as are accurate photoelectric $V$ and $B-V$ values. A new calibration of the MK absolute magnitudes has been employed in calculating distances. The new absolute magnitudes have been derived from stars that have known MK spectral types and luminosity classes and that are members of clusters whose distances are known from accurate $V, B-V$ photometry. The distance scale of the stellar population under discussion is thus consistent with the cluster distance scale which, in turn, is ultimately based upon the kinematic distance scale of the Hyades.

\section{A Simple Test for Radial Motions}

We postulate that the expected motion of any object can be described in terms of circular motion, $\omega(R)$, and galactic radial motion, $E(R)$. Neither function depends upon $\theta$; we assume that all objects studied lie so near to the galactic plane that we can neglect the difference between $\omega(R, Z)$ and $\omega(R, 0) \equiv \omega(R)$ and between $E(R, Z)$ and $E(R, 0) \equiv E(R)$. The local standard of rest is representative of galactic motion $\omega\left(R_{0}\right) \equiv \omega_{0}, E\left(R_{0}\right) \equiv E_{0}$. Radial velocities measured with respect to the local standard of rest are denoted by $v_{r}^{\prime}$. The expectations of these measured radial velocities arise from differential galactic motions and are specified by the equations:

$$
v_{r}^{\prime}=R_{\mathrm{o}}\left[\omega(R)-\omega_{0}\right] \sin l \cos b+E\left(R_{0}\right) \cos l \cos b-E(R) \cos (l+\theta) \cos b,
$$


from which

$$
\frac{v_{r}^{\prime}}{\sin l \cos b}=R_{0} \Delta \omega(R)+E_{0} \cot l-E(R) \frac{\cos (l+\theta)}{\sin l}
$$

or

$$
\frac{v_{r}^{\prime}}{\sin l \cos b}=R_{0} \Delta \omega(R)+\frac{\Delta E(R, l)}{\sin l} .
$$

The functions $\Delta \omega(R)$ and $\Delta E(R, l)$ in equation (3) differ in character. The difference $\Delta \omega(R)$ is strictly $\omega(R)-\omega_{0}$, but the difference $\Delta E(R, l)$ is a quantity that involves not only $E(R)$ and $E\left(R_{\theta}\right)$ but also contains projection factors; it is not independent of $l$ or, alternatively, of $\theta$.

The observable $v_{r}^{\prime} /(\sin l \cos b)$ in equation (3) is the quantity most advantageously employed in the study of radial motion in the Galaxy; it provides a particularly powerful means of investigating $\Delta E(R, l)$ at "corresponding points" in the Galaxy. Corresponding points in the galactic plane are points which have

(i) identical $R$ values, and

(ii) $l$ values (or $\theta$ values) that are equal in absolute value but opposite in sign. At corresponding points in the Galaxy:

(i) $R_{0} \Delta \omega(R)$ values are identical;

(ii) $\Delta E(R, l)$ values are identical;

(iii) $\sin l$ values are the same numerically but opposite in sign.

Because of these facts

$$
\left(\frac{v_{r}^{\prime}}{\sin l \cos b}\right)_{\text {point } 1}-\left(\frac{v_{r}^{\prime}}{\sin l \cos b}\right)_{\text {point } 2}=2^{\prime}\left(\frac{\Delta E(R, l)}{\sin l}\right)_{\text {point } 1} .
$$

By taking differences of $v_{r}^{\prime} /(\sin l \cos b)$ values at corresponding points, we eliminate the rotational component of the Galaxy from the resulting equation and double the $\Delta E(R, l)$ component.

With the observed quantities $v_{r}^{\prime} /(\sin l \cos b)$ for the sample population of young stars, we make two simple preliminary tests to determine if there is any differential galactic radial component in their motions.

(i) In Figure $1(a)$ we compare $v_{r}^{\prime} /(\sin l \cos b)$ (plotted as a function of $R$ ) for all stars in quadrant I with $v_{r}^{\prime} /(\sin l \cos b)$ (plotted as a function of $R$ ) for all stars in quadrant IV. It is notable that the points representing stars in quadrant IV (filled circles) lie systematically above the points representing stars in quadrant I (open circles).

(ii) We compare, over a restricted $\Delta R$ range in which the stellar density is reasonably high, a plot of $v_{r}^{\prime} /(\sin l \cos b), R$ for stars in quadrant II and for stars in quadrant III. (See Fig. $1(b)$.) At any one $R$-value $R_{0} \omega(R)$ has the same numerical value whatever the value of $l$. However, we find, for identical $R$-values, different values of $v_{r}^{\prime} /(\sin l \cos b)$ for objects in quadrants II and III.

The tests in (i) and (ii) both indicate a non-zero value of $\Delta E(R)$. The results obtained from the tests are of the nature expected from galactic expansion following a law, for example, of the form $E(R) \propto R^{-n}$ or of galactic contraction, for example, of the general form $E(R) \propto-R^{n}$. We therefore proceed to investigate the observed 
velocities in greater detail to determine if they can be represented by a combination of smooth functions representing galactic rotation and galactic radial motion.

\section{Investigation of a Model Galaxy}

If galactic rotation $\omega(R)$ and radial motion $E(R)$ are present, the expectation of a star's radial velocity is given by equation (1), which we rewrite as

$$
\frac{v_{r}^{\prime}}{\sin l \cos b}=R_{0} \Delta \omega(R)+E_{0} \cot l-E(R) \frac{\cos (l+\theta)}{\sin l} .
$$

If we represent $\omega(R)$ by a linear function and, with Kerr, represent $E(R)$ by a power law $k / R^{n}$, we find, from equation (5),

$$
\frac{v_{r}^{\prime}}{\sin l \cos b}=R_{0} \omega_{0}^{\prime} \Delta R+E_{\mathrm{o}}\left[\cot l-\left(\frac{R_{\mathrm{o}}}{R}\right)^{n} \frac{\cos (l+\theta)}{\sin l}\right] \text {. }
$$
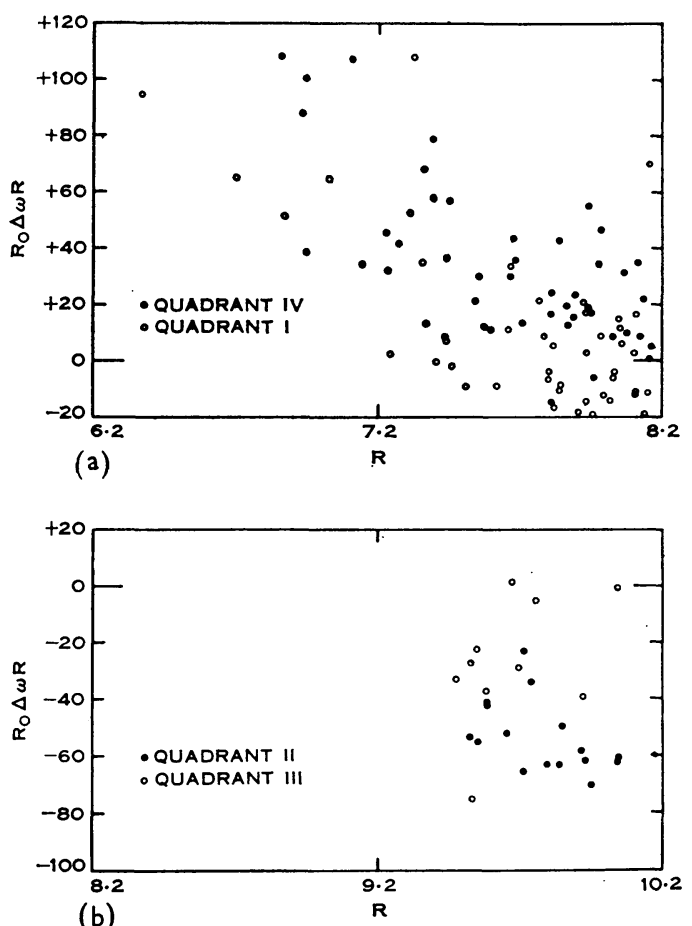

Fig. 1(a).-Plot of $v_{\mathbf{r}}^{\prime} /(\sin l \cos b)$ (also called $\left(R_{0} \Delta \omega(R)\right.$ ) for all stars in quadrants $I$ and IV.

Fig. $1(b)$. - Plot of $v_{\mathrm{r}}^{\prime} /(\sin l \cos b)$ for stars in quadrants II and III lying within a limited $\Delta R$-range.

If $n=1$ in equation (6), the equation states that the expansion takes place freely at such a rate that in unit time interval the same amount of gas flows across any circular boundary centred on the galactic centre and lying in the galactic plane. A value of $n>1$ signifies that the expanding gas experiences deceleration through collision processes or from other causes. 
Equation (6) is of interest since it involves both kinematic conditions (through $v_{r}, \omega_{0}^{\prime}, E_{0}$, and $n$ ) and geometric conditions (through $l, b, R_{0}, \Delta R$, and $\theta$ ). In principle we can use equation (6) to estimate numerical values of both the kinematic parameters and the galactic scale value $R_{0}$. The method of making such a solution can best be illustrated by reference to a simple galactic model which should, at the same time, provide an indication of the behaviour of parameter values we should expect to find in the real Galaxy.

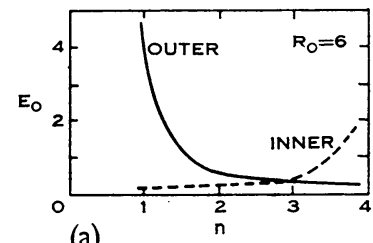

(a)

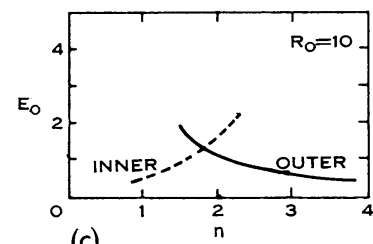

(c)

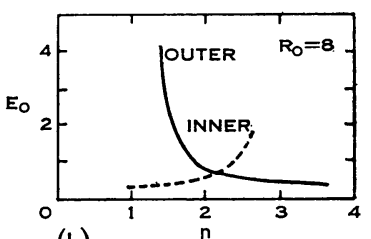

(b)

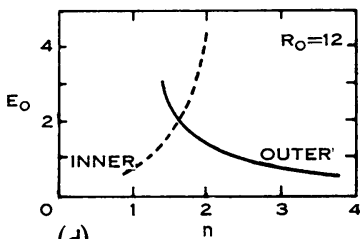

(d)

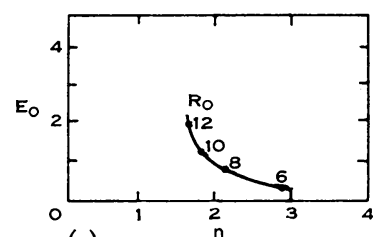

(e)

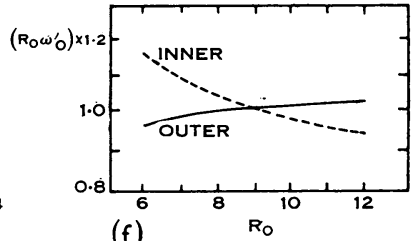

(f)

Fig. 2.-Model galaxy results. $(a),(b),(c),(d)$ : Plots of $E_{0}$ as a function of $n$ for different values of $R_{0}$. In each of these diagrams the unit of $E_{0}$ (i.e. the value of $E_{0}=1$ ) is the true value of $E_{0}$ in our adopted model galaxy in which $R_{0}=9 \mathrm{kpc}$. Thus $E_{0}$ is expressed in consistent units whatever the assumed value of $R_{0}$. (e): Plot of the solving $E_{0}, n$ pairs as a function of $R_{0}$. The solving $E_{0}, n$ pair for any $R_{0}$ value is the cross-over point of the inner and outer curves in each of the plots $(a),(b),(c),(d)$. $(f)$ : Plot of $R_{0} \omega_{0}^{\prime}$ as a function of $R_{0}$ shown for the inner and outer star groups individually. The unit on the $R_{0} \omega_{0}^{\prime}$ axis is the true value of $R_{0} \omega_{0}^{\prime}$ in our adopted model galaxy for which $R_{0}=9 \mathrm{kpc}$.

In our model galaxy we place ourselves at $R_{0}=9 \mathrm{kpc}$; all objects move in circular orbits in accord with the expression $\omega(R)=\omega_{0}+\omega_{0}^{\prime} \Delta R$ and experience radial motion $E(R)=E_{0}\left(R_{\mathrm{o}} / R\right)^{n}$. For a sample population of stars we measure $v_{r}^{\prime}, l, b$, and $r$. We wish to determine $R_{0}, R_{0} \omega_{0}^{\prime}, E_{0}$, and $n$. To do so we assign a series of values to $R_{0}$ and $n$ and proceed to derive values of $R_{0} \omega_{0}^{\prime}$ and $E_{0}$ for each assigned $R_{0}, n$ pair. The geometrical aspects of the equation are brought into the problem by dividing the sample population into two parts - the part of the population that lies in the inner quadrants, I and IV, and that part of the population that lies in the outer quadrants, II and III - and deriving the values of $R_{0} \omega_{0}^{\prime}$ and $E_{0}$ for each of these sub- 
populations. We shall find that $R_{\mathrm{o}} \omega_{\mathrm{o}}^{\prime}$ is almost independent of $n$ for any $R_{\mathrm{o}}$ value (it does depend slightly upon the spatial distribution of the population) and that for any $R_{0}$ value only one value of $n$ will satisfy both the outer and inner quadrant populations. Figure 2 shows the mathematical results providing the basis for these remarks.

For our model galaxy our procedure recovers the correct $R_{0} \omega_{0}^{\prime}, R_{0}$ pair from the rotation curve, and provides a family of $E_{0}, n$ values. We can, of course, use the $R_{0}$ value derived from $R_{0} \omega_{0}^{\prime}, R_{0}$ to infer the value of $E_{0}$. If our model galaxy possesses an observable $3 \frac{1}{2}-\mathrm{kpc}$ expanding arm as the real Galaxy does, we can make use of the data from that arm to obtain a second curve in the $E_{0}, n$ plane and in this manner derive the true $E_{0}, n$ value independently of the $R_{0} \omega_{0}^{\prime}, R_{0}$ pair.

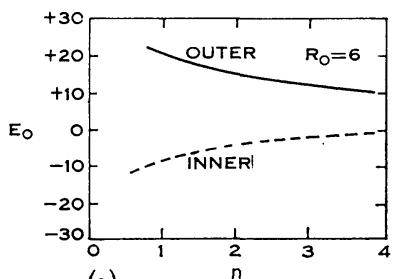

(a)

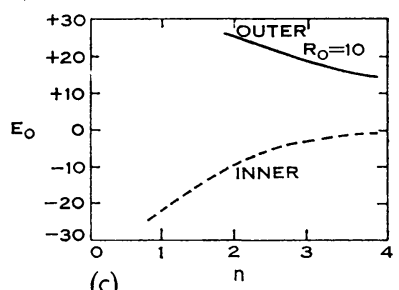

(c)

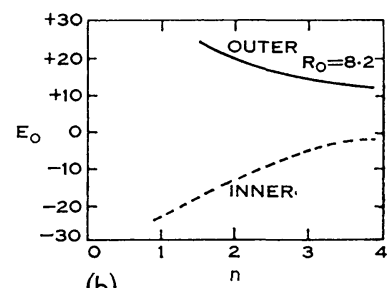

(b)

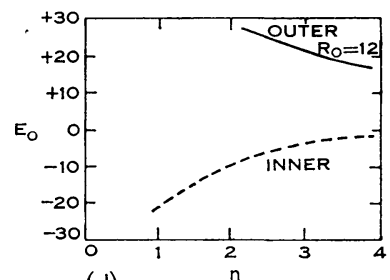

(d)

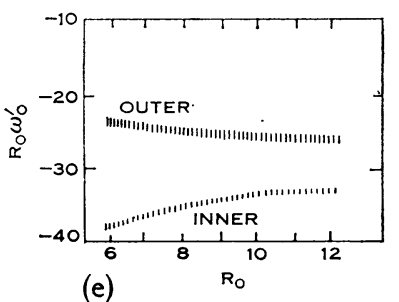

(e)

Fig. 3.-Real Galaxy results. $(a),(b),(c),(d):$ Plots of $E_{0}$ (in $\left.\mathrm{km} / \mathrm{sec}\right)$ as a function of $n$ for different values of $R_{0}$. (e): Values of $R_{0} \omega_{0}$ (in $\mathrm{km} / \mathrm{sec} . \mathrm{kpc}$ ) as a function of $R_{0}$ shown for the inner and outer groups of stars separately. The width of the cross-hatched lines show the sensitivity of $R_{0} \omega_{0}^{\prime}$ to the value of $n$.

The distance of the $3 \frac{1}{2}-\mathrm{kpc}$ arm has been found by locating the point of tangency of the line of sight, which gives, for the expanding arm, the ratio $R_{\mathrm{o}} / R=\mathbf{2} \cdot \mathbf{3 9}$, and hence the observed velocity of expansion for the $3 \frac{1}{2}-\mathrm{kpc}$ arm $=\left[(2 \cdot 39)^{n}-1\right] E_{0}$ which provides a second $E_{0}, n$ relation. Unfortunately, the $\left(E_{0}, n\right)$ curves from the stars and the expanding arm osculate. They do not cross and thus provide a strong determination of the correct $\left(E_{0}, n\right)$ pair. 
We now treat the real Galaxy as we did the model in an effort to derive the kinematic parameters and the scale of the Galaxy.

\section{Results for the Real Galaxy}

Figures $3(a),(b),(c),(d)$ exhibit the $\left(E_{0}, n\right)$ relation derived from the OB stars in the Galaxy for different $R_{0}$ values. Figure $3(e)$ shows the $\left(R_{0} \omega_{0}^{\prime}, R_{0}\right)$ relation for the same stars. In this last diagram the widths of the lines indicate the extent of the dependence of $R_{0} \omega_{0}^{\prime}$ upon $n$.

The results for the real Galaxy do not resemble those computed for the model. In particular, no $\left(E_{0}, n\right)$ curves cross and the $\left(E_{0}, n\right)$ relation for stars in the inner quadrants exhibits a negative $E_{0}$-value. There is likewise no intersection of the $\left(R_{0} \omega_{0}^{\prime}, R_{0}\right)$ curves. Though our first tests showed radial motion to be present, the kinematic model we adopted for the Galaxy, a model involving circular motion and a power law for expansion, does not fit the very young stars. We therefore reject equation (6) as representative of the sample population of $\mathrm{OB}$ stars and proceed to derive the empirical galactic radial motion field exhibited by the sample population.

\section{An Empirical Galactic Radial Motion Field}

The lack of intersection of the $\left(R_{0} \omega_{0}^{\prime}, R_{0}\right)$ curves for the inner and outer quadrants implies that a linear relation is inadequate for $\Delta \omega(R)$ and that we must adopt a form $\omega(R)=\omega_{0}+\omega_{0}^{\prime} \Delta R+\frac{1}{2} \omega_{0}^{\prime \prime}(\Delta R)^{2}$ to represent the sample population as

TABLE 1

ROTATIONAL PARAMETERS FOR OB STARS

\begin{tabular}{c|c|c}
$R_{0}$ & $\begin{array}{c}R_{0} \omega_{0}^{\prime} \\
(\mathrm{kpc})\end{array}$ & $\begin{array}{c}\frac{1}{2} R_{0} \omega_{0}^{\prime \prime} \\
\left(\mathrm{km} / \mathrm{sec} . \mathrm{kpc}^{2}\right)\end{array}$ \\
\hline $6 \cdot 0$ & $-30 \cdot 80$ & $+3 \cdot 68$ \\
$8 \cdot 2$ & $-28 \cdot 82$ & $+1 \cdot 56$ \\
$10 \cdot 0$ & $-28 \cdot 33$ & +0.99 \\
$12 \cdot 0$ & -27.99 & +0.67 \\
\hline
\end{tabular}

a whole. As an alternative to such a quadratic expression we can adopt the hypothesis that there are regional systematic motions in the rotational as well as in the radial direction. This implies that we may expect regional variations in observed $\omega(R)$ values as well as in radial motions. For the present, we reject this latter hypothesis and assert that there is a quadratic expression for $\omega(R)$ by which we can represent the rotation component. On this basis we proceed to derive the empirical radial motion field.

Experience as well as theory shows that numerical values of $R_{0} \omega_{0}^{\prime}$ and $\frac{1}{2} R_{0} \omega_{0}^{\prime \prime}$ are little affected by the radial-motion term in equation (5). For discussion of $R_{\mathrm{o}} \Delta \omega(R)$ we therefore set $E(R)=0$ and treat the entire population simultaneously to find the rotational parameter values shown in Table 1. 
With these values we compute the galactic radial motion field with the aid of the equation

$$
\left[\frac{v_{r}}{\sin l \cos b}-R_{0} \omega_{0}^{\prime} \Delta R-\frac{1}{2} R_{0} \omega_{0}^{\prime \prime}(\Delta R)^{2}\right] \sin l=\Delta E(R, l) .
$$

In a galaxy in which there is large-scale systematic radial motion, we expect the quantity $\Delta E(R, l)$ to be symmetrical about the centre-anticentre line; $\Delta E(R, l)$ $=-\Delta E(R,-l)$.

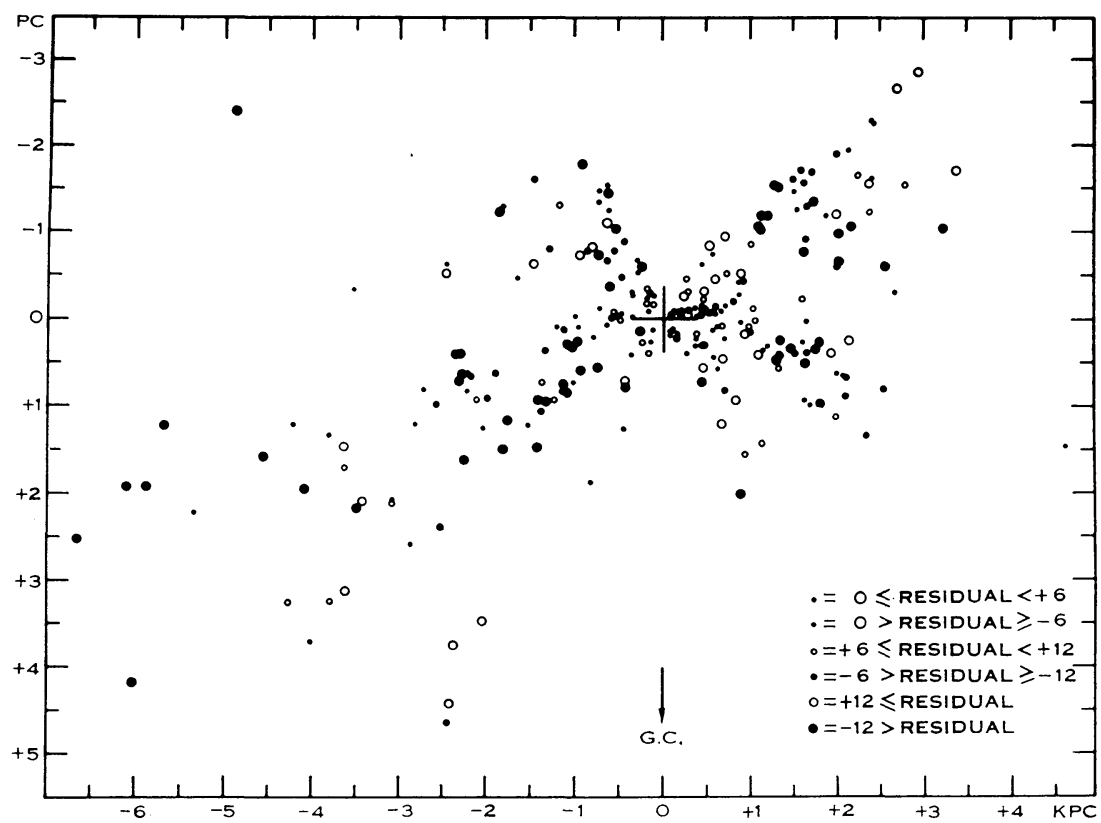

Fig. 4.-A plot of residuals, $\Delta E(R, l)$, for individual stars among the population of very-early-type stars examined in this investigation. evident.

The empirical velocity field $\Delta E(R, l)$ is shown in Figure 4. Two features are

(i) The expected symmetry about the $0-180^{\circ}$ line is not present.

(ii) The empirical velocity field is irregular. Positive values of $\Delta E(R, l)$ will be found principally in one restricted region of the Galaxy, large negative values in another restricted region, small negative values in another.

Because of these large-scale regional motions, the very young stars for which data are currently available provide neither a proof nor a denial of the expansion law proposed by Kerr. The existence of large-scale regional peculiar motions among the very young stars has an important implication for our observational investigation of the Galaxy: if a general field of expansion velocities does exist and if we are to derive numerical values of the parameters of that field from the very young stars, we must observe the velocity field of the very young stars over a volume element of the Galaxy of such size that it contains a significant number of large-scale motion elements. Only in this way can we obtain meaningful average values of the radial- 
motion parameters of the Galaxy. The volume element containing the stellar population with which we have been concerned in this investigation does not contain a sufficient number of such elements to permit us to obtain a satisfactory general radialmotion law; it is of sufficient size only to indicate the large-scale irregularities that appear to exist.

\title{
VIII. The Differential Rotation Parameter
}

As a final remark about the properties of the field of motion of the very young stars we give in Table 2 , as a function of $R_{0}$, the $A$-values derived directly from the parameters listed in Table 1.

\section{TABLE 2}

VALUES OF THE $A$-PARAMETER OF GaLACTIC ROTATION DERIVED From THE VERY YOUNG STARS AND SHOWN AS A FUNCTION OF THE DISTANCE TO THE GALACTIC CENTRE

\begin{tabular}{|c|c|c|c|}
\hline $\begin{array}{c}R_{0} \\
(\mathrm{kpc})\end{array}$ & $\begin{array}{c}A=-\frac{1}{2} R_{0} \omega_{0}^{\prime} \\
(\mathrm{km} / \mathrm{sec} \cdot \mathrm{kpc})\end{array}$ & $\begin{array}{c}R_{0} \\
(\mathrm{kpc})\end{array}$ & $\begin{array}{c}A=-\frac{1}{2} R_{0} \omega_{0}^{\prime} \\
(\mathrm{km} / \mathrm{sec} . \mathrm{kpc})\end{array}$ \\
\hline 6 & $15 \cdot 4$ & 10 & $14 \cdot 2$ \\
\hline $8 \cdot 2$ & $14 \cdot 4$ & 12 & $14 \cdot 0$ \\
\hline
\end{tabular}

\section{Discussion}

Kerr: It is interesting to see a clumpiness in the stellar velocities similar to that of the gas. What is the average area covered by one of these "clumps"?

Weaver: The region of Perseus, for example, is about $800 \mathrm{pc}$ wide and about $1.2 \mathrm{kpc}$ long. It may possibly be longer but we have no data. The other regions are generally about $1 \mathrm{kpc}$ in diameter.

Feast: Is it true to say that you have used the known associations as single points in your plot?

Weaver: Yes, associations, clusters, and single stars were considered as single points. This is because the peculiar motion of a cluster is about the same as that of a single star. For clusters, of course, the distance is generally better determined.

\section{A HIGH-RESOLUTION STUDY OF M31}

\author{
B. F. Burke, K. C. Turner, and M. A. Tuve
}

Carnegie Institution of Washington, Department of Terrestrial Magnetism

The great nebula in Andromeda, M31, is a particularly interesting external galaxy, since it is a giant spiral system, presumably much like our own Galaxy, and is close enough to be resolved with existing radio telescopes. During parts of November 1962, and from December 5, 1962, to January 20, 1963, the 300-foot transit telescope of the N.R.A.O. at Green Bank, W.Va., was used in conjunction with the Carnegie multichannel $\mathrm{H}$-line spectrograph to study M31. The following is a preliminary account of these first observations. 